\title{
Brustkrebs: Eine Anziehpuppe erleichtert Gespräche zwischen Eltern und Kindern
}

Sabine Jenny

Projektleitung,

Krebsliga Schweiz
Korrespondenz:

Krebsliga Zentralschweiz Hirschmattstrasse 29

CH-6003 Luzern

Tel. 0412102550

info[at]krebsliga.info

www.krebsliga.info
Krebskranke Eltern oder Verwandte, die unsicher sind, wie sie mit Kindern über eine Krebserkrankung sprechen sollen, konsultieren häufig ihren Arzt oder ihre Ärztin mit der Bitte um Unterstützung. Mit der Illustrationspuppe «Madame Tout-Le-Monde» bietet die Krebsliga neu ein hilfreiches Instrument an, um mit jüngeren Kindern ins Gespräch zu kommen, ihnen «Brustkrebs» verständlich zu machen und sie zu ermuntern, Fragen zu stellen. Entwickelt wurde die Illustrationspuppe von Fabienne Roth Duss, die 2011 selber vor der Situation stand, ihre beiden Töchter im Kleinkindalter mit ihrer BrustkrebsErkrankung konfrontieren zu müssen.

\section{Ein Bastelbogen und ein Computerspiel machen Mut}

Das Prinzip der Puppe ist einfach: Als Projektionsfläche soll sie helfen, die Situation, die derzeit für das Kind in seinem Alltag mit der kranken Mutter aktuell ist, zu thematisieren. Dabei gilt es, den momen-

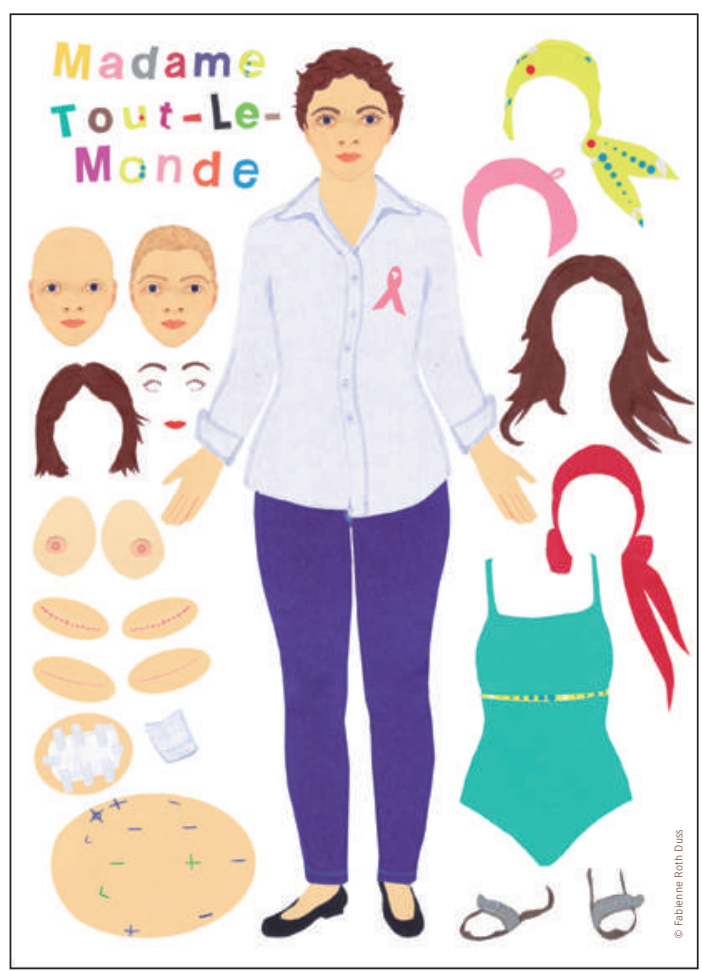

Die Illustrations-Mappe mit Puppe und Anleitung kann von Ärzten und Pflegenden bezogen und kostenlos an Betroffene abgegeben werden. Bestellungen: Krebsliga Schweiz, Kundendienst/Versand, Postfach 110,

3766 Boltigen; shop[at]krebsliga.ch; www.krebsliga.ch/ broschueren; www.madame-tout-le-monde.ch

\begin{abstract}
Brustkrebs-Monat Oktober: «Gemeinsam um die Welt»

Im Oktober möchte die Krebsliga anlässlich des InfoMonats-Brustkrebs ein besonderes Zeichen der Solidarität setzen und gemeinsam mit vielen Menschen ein Mal die Welt umrunden. Das entspricht $40000 \mathrm{~km}$, die spazierend, joggend oder per Velo zurückzulegen sind. Organisieren Sie im Oktober mit Freunden oder Kollegen einen Lauf oder eine Velotour. Auch als Unternehmen können Sie sich an der Aktion beteiligen. Bestellen Sie Flyer und rosa Ansteckschleifen oder unseren Thekensteller, der bereits mit 50 Flyern und 50 rosa Schleifen bestückt ist. Als Botschafterinnen engagieren sich die Triathletin Nicola Spirig, die Snowboarderin Tanja Frieden, die Rollstuhl-Athletin Edith Hunkeler, die Schwimmerin Swann Oberson und die Hochspringerin Beatrice Lundmark. Mehr Informationen: www.krebsliga.ch/brustkrebs
\end{abstract}

tanen Krankheits- und Therapieverlauf auf der Puppe abzubilden. «Madame Tout-Le-Monde» wechselt ihre Kleider und verändert ihr Haar, hat einen kahlen Kopf, trägt bunte Kopftücher und Mützen. Es können ihr Verband und Prothesen angeheftet und mit ihr Chemo- und Radiotherapie erklärt werden. Natürlich kann man Positives vorwegnehmen. Dann versieht man «Madame Tout-Le-Monde» einfach mit einer Kurzhaarfrisur, um zu zeigen, dass die Haare später, nach der Chemotherapie, wieder nachwachsen werden. Analog zur Papierpuppe steht auch eine Website als virtuelles Puppenspiel zur Verfügung. «Madame Tout-Le-Monde» macht Mut und gibt Hoffnung, ohne zu beschönigen.

Ärztinnen und Ärzte sowie andere Fachpersonen, die mit Brustkrebsbetroffenen zu tun haben, können die Bastelmappe bei der Krebsliga oder auf der Projektwebsite bestellen, um sie kostenlos an Betroffene weiterzugeben: Die Mappe umfasst verschiedene A3-Bastelbögen. Gemeinsam mit dem Kind können Figuren und Kleider ausgeschnitten, besprochen und bemalt werden. Das Online-Spiel enthält neben der Welt der «Madame Tout-LeMonde» weitere Beratungs- und Unterstützungsangebote. Zum Brustkrebsmonat Oktober 2013 wird das Angebot um eine App ergänzt. 2014 soll das Angebot auf Französisch und Italienisch vorliegen. 